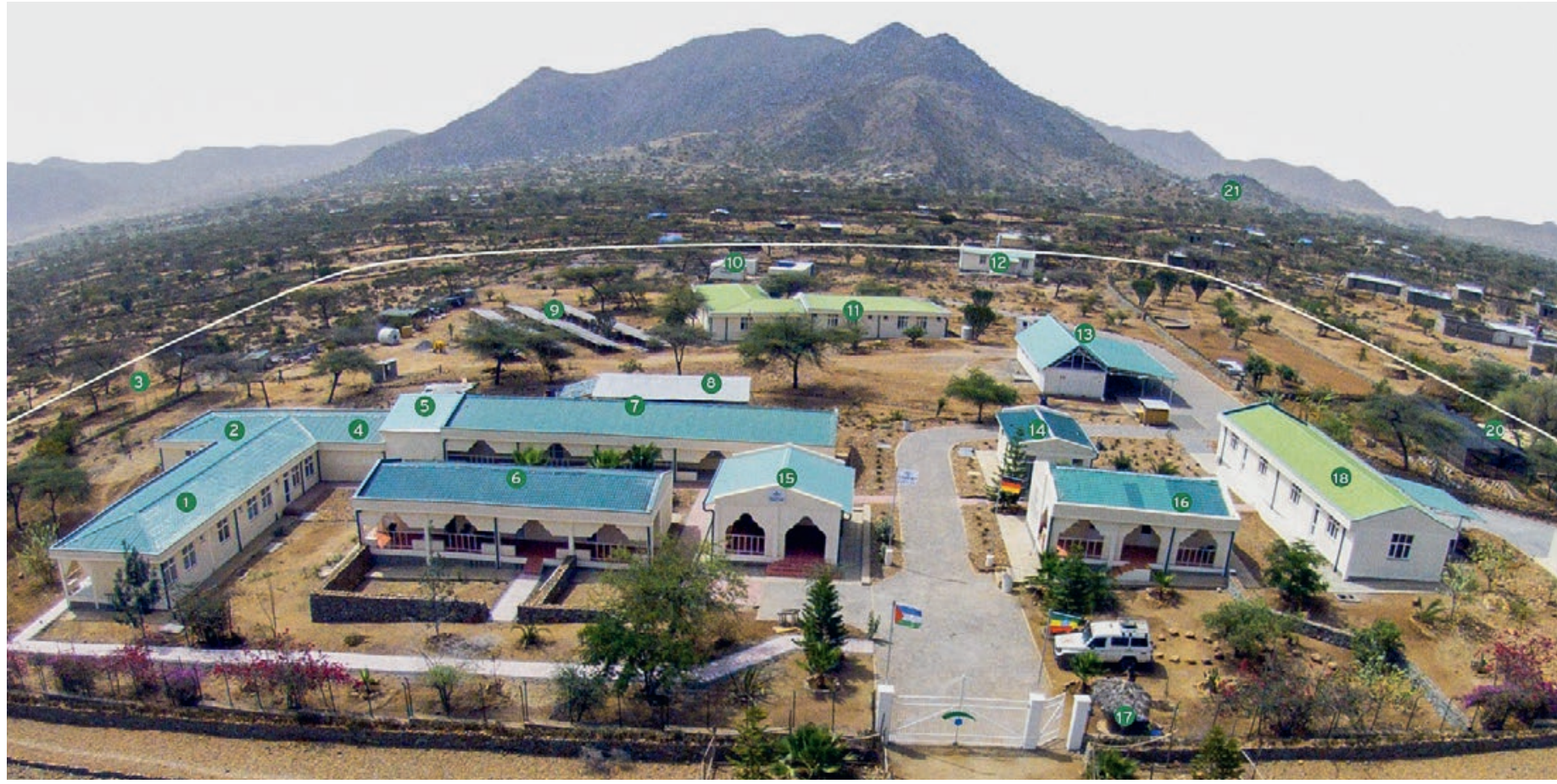

Das Klinikareal in der Danakilwüste. 1: Patientinnen stationär; 2: Operationssäle; 3: Kläranlage/Verbrennung; 4: Sterilisation; 5: Lagerräume; 6: Mitarbeiterhaus; 7: Entbindungsräume / Gynäkologische Ambulanz / Labor; 8: Lager Baumaterialien; 9: Photovoltaikfeld; 10: Container für künftigen Wohnraum Mitarbeiter; 11: Wohnhaus medizinische Mitarbeiter; 12: Mitarbeiterhaus; 13: Versorgungshaus aus Containern: Wasseraufbereitung / Batterien PVA /

Werkstatt / Wohnraum Mitarbeiter; 14: Duschen/WC; 15: Empfang; 16: Mitarbeiterräume; 17: Wächterhäuschen (im Bau); 18: Küche/Wäscherei/Mitarbeiterkantine; 19: Generator; 20: Gemüsegarten; 21: Brunnen

\title{
Oase der Hoffnung
}

\section{Tanja Kühnle}

Dipl.-Biol., Managing Editor SÄZ

Ihr Kind sicher und geborgen auf die Welt bringen - lange ein unerfüllbarer Wunsch für die Frauen der Afar, die nahezu alle genital verstümmelt sind. Eine natürliche Geburt ist in solchen Fällen lebensgefährlich für Mutter und Kind. Doch medizinische Hilfe war für das Halbnomadenvolk in der Wüste Äthiopiens lange nicht in Sicht. Annette und Rüdiger Nehberg kämpfen mit ihrer Menschenrechtsorganisation TARGET e.V. seit vielen Jahren unermüdlich für das Ende der weiblichen Genitalverstümmelung und haben 2015 am Rand der Danakilwüste eine Klinik für Gynäkologie und Geburtshilfe eröffnet. Seither haben hier mehr als 1000 Babys das Licht der Welt erblickt - auch mit Unterstützung von Ärzten aus der Schweiz.

«Manchmal fühlten wir uns wie Pyramidenbauer mit all diesen unglaublichen Herausforderungen", erinnert sich Projektleiter Roman Weber an die fast fünfjährige Bauzeit der Klinik am Rand der äthiopischen Wüste. «Doch wir hatten das Ziel immer vor Augen: den Mädchen und Frauen Hilfe zu bringen, die hier bitter nötig ist.»
Eine genaue Statistik, wie viele Mädchen und Frauen weltweit von weiblicher Genitalverstümmelung (FGM, engl. female genital mutilation, siehe Kasten) betroffen sind, gibt es nicht. Laut UNICEF sind aktuell von dem grausamen Ritual mindestens 200 Millionen Mädchen und Frauen betroffen [1]. Ihre Leiden sind unvorstellbar schlimm. 


\section{FGM - was ist das?}

Weibliche Genitalverstümmelung (engl. female genital mutilation, kurz FGM) bezeichnet nach einer Definition der Weltgesundheitsorganisation WHO die partielle oder vollständige Entfernung der äusseren weiblichen Genitalien [4]. Die Verstümmelung erfolgt in vielen Ländern aus traditionellen oder kulturellen Motiven und ist vor allem in islamisch geprägten Gebieten Afrikas weit verbreitet. Sie wird oftmals - fälschlicherweise - mit der heiligen Schrift des Koran begründet bzw. gerechtfertigt.

In vielen Ländern Afrikas ist FGM ein festes Ritual, das an Mädchen im Alter von wenigen Wochen bis zur Pubertät sowie an Frauen vollzogen wird. So sollen die Mädchen vor ihrer eigenen Sexualität "geschützt» und als «rein» bewahrt werden, damit der zukünftige Ehemann die Frau als jungfräulich anerkennt. Ohne den Akt der Verstümmelung droht den Mädchen bzw. Frauen, von der Gemeinschaft verstossen zu werden.

Durchgeführt wird die Verstümmelung von sogenannten «Beschneiderinnen», meist älteren Frauen, die das Ritual seit vielen Jahren vollziehen und dafür oft bezahlt werden. Als «Werkzeuge» dienen Messer und Rasierklingen. Um die Wunde zu "vernähen» und die Beine nach der Verstümmelung zusammenzuhalten, werden Akaziendornen und Schlingen verwendet. Bei der schlimmsten Form, der sogenannten pharaonischen Verstümmelung, wird zudem noch ein Strohhalm verwendet, um dem Opfer eine kleine Öffnung für Urin und die Menstruationsblutung zu erhalten.

Im Afar-Gebiet, wo die "pharaonische Verstümmelung» (Typ III der WHO-Klassifikation [2]) als traditionell schlimmste Form weit verbreitet ist, liegt die Säuglingssterblichkeit ohne medizinische Versorgung nach Aussagen der örtlichen Hebammen bei 50\%. Der Grund: Durch die Verstümmelung ist der Geburtsweg teilweise verschlossen und wegen der Narben nicht ausreichend elastisch. Für einen Kaiserschnitt mussten die Frauen jedoch in weit entfernte Städte gebracht werden - das war nur wenigen von ihnen möglich. Deshalb hat sich TARGET e.V. im Jahr 2010 dazu entschlossen, eine Klinik am Rand der Danakilwüste beim Volk der Afar zu bauen.

Und so haben viele fleissige Helfer in einer der heissesten Regionen der Erde ohne Baumaschinen, Lastwagen und Infrastruktur, dafür mit viel Handarbeit, grossem persönlichem Engagement und noch mehr Herzblut, ein weitgehend autarkes Klinikareal von hohem medizinischem Qualitätsstandard geschaffen: Es gibt Ent- bindungsräume, Operationssäle, einen Patiententrakt mit zehn Betten, ein Labor, eine Wäscherei, eine Sterilisation, eine Küche und einen Gemüsegarten für die Selbstversorgung, ein Ärzte- und ein Mitarbeiterwohnhaus, eine Apotheke, eine Klär- und eine Verbrennungsanlage sowie ein Photovoltaikfeld zur Stromgewinnung. Die medizinischen Geräte und das Inventar sind grösstenteils Sachspenden aus Kliniken.

Mittlerweile arbeiten in der Klinik mehr als 50 Men schen in einem vorwiegend deutsch-äthiopischen Team aus Medizinern, Krankenschwestern und Hebammen, Technikern, Küchen- und Reinigungspersonal, Ambulanzfahrern, Wachmännern und Verwaltungsangestellten - sie alle eint der Kampf gegen FGM. Um die fachliche Betreuung zu gewährleisten, hat TARGET e.V. noch vor der Planung des Projekts eine Kooperation mit dem Berufsverband der Frauenärzte e.V. (BVF) in Deutschland vereinbart. Seither haben viele Mediziner aus Deutschland, Österreich und der Schweiz das Projekt vor Ort unterstützt. Eine von ihnen ist Dr. Roswitha Hausdorf, Fachärztin für Gynäkologie und Geburtshilfe in Schwyz. Sie war durch einen Beitrag in der Fachzeitschrift Frauenarzt auf das Projekt in der Danakilwüste aufmerksam geworden.

\section{Auch für Mediziner eine belastende Situation}

Roswitha Hausdorf war im Sommer 2017 für insgesamt sechs Wochen vor Ort im Einsatz. An ihre erste Geburt in der Wüstenklinik erinnert sie sich sehr genau: «Die Patientin hatte eine pharaonische Beschneidung, Typ III in der WHO-Klassifikation. Bei dieser Form werden von traditionellen Hebammen mit einfachsten Werkzeugen wie Rasierklingen, Dosendeckeln oder Scherben ohne jede Betäubung die sichtbare Klitoris,

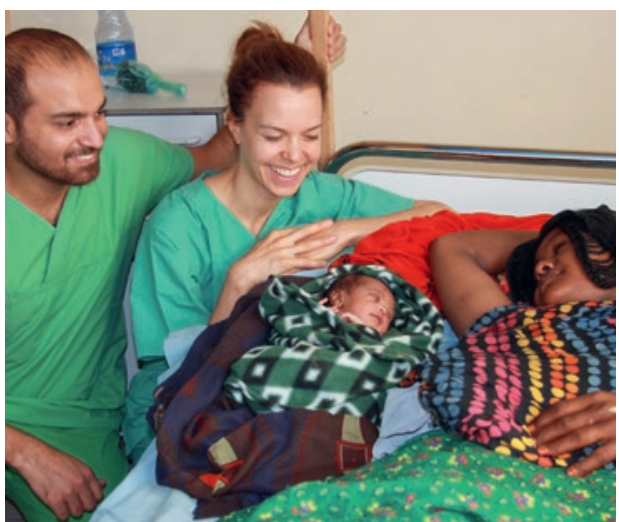

Geburt geglückt: Majid Dawd (links) und Kollegin Flora Graefe am Bett von Mutter und Kind.

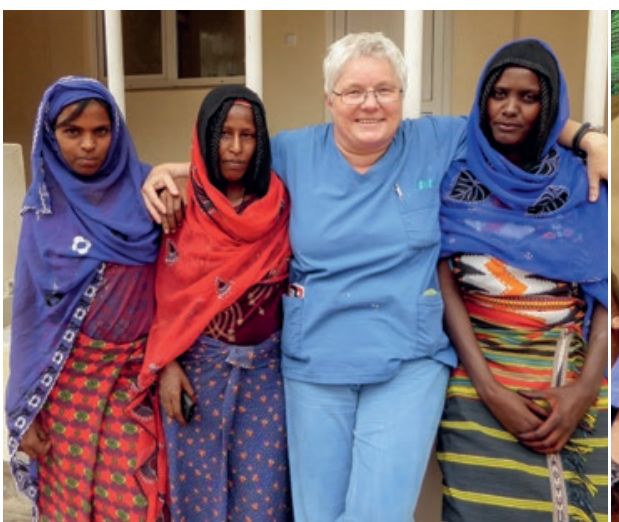

Roswitha Hausdorf zusammen mit drei einheimischen Hebammen.

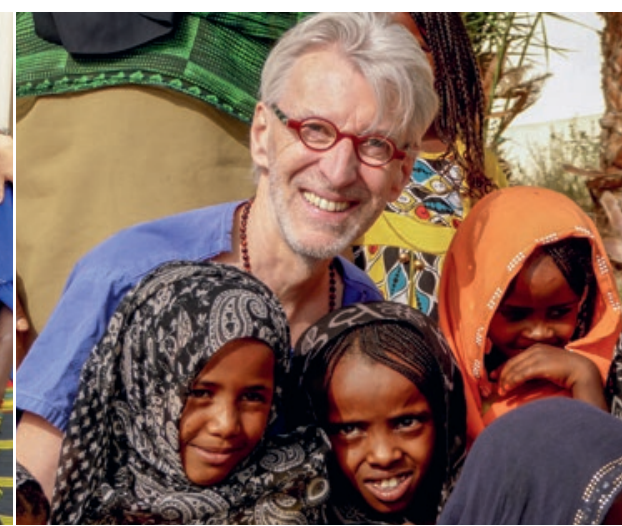

Unermüdlich im Einsatz: Ludwig Kronpaß reist schon zum dritten $\mathrm{Mal}$ in die Danakilwüste. 


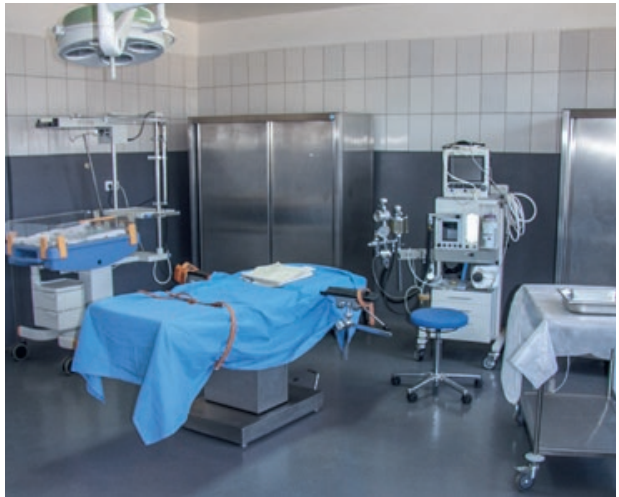

Ein Blick in den Operationssaal.

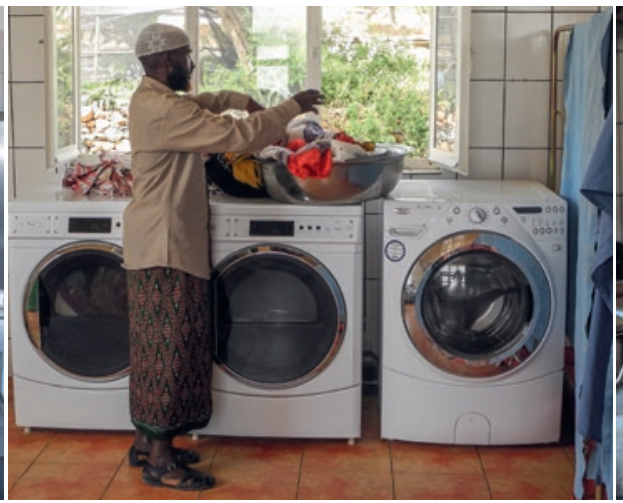

Saleh ist zuständig für die Wäscherei.

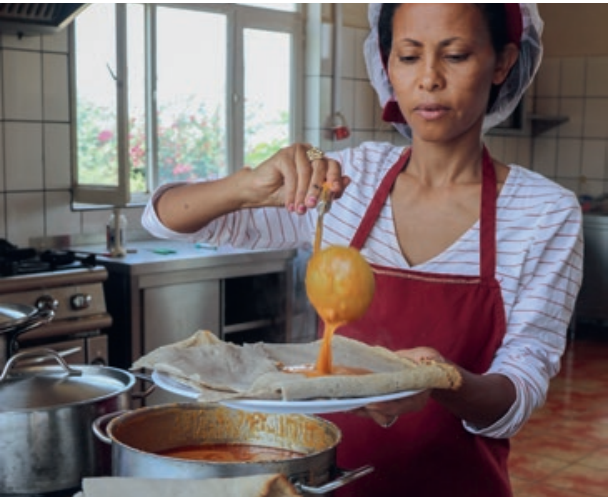

Küchenchefin Fotein versorgt die Patientinnen mit typisch äthiopischem Essen. ermutigen die Frauen und ihre Familien dazu, schon vor der Geburt zur Schwangerschaftsvorsorge in die Klinik zu kommen. So können die Geburten geplant, manchmal direkt ein Kaiserschnitt empfohlen werden. Viele schwerwiegende Komplikationen werden so vermieden.

\section{Nachsorge ist Vorsorge}

Auch auf die nachgeburtliche Versorgung der Frauen wird in der Klinik grossen Wert gelegt. Denn während es in dieser Gegend normalerweise üblich ist, die Vagina nach der Entbindung wieder zuzunähen und den Frauen so erneut grosses Leid zuzufügen, geht die Organisation von Annette und Rüdiger Nehberg einen anderen Weg: Werdende Eltern, die in der Klinik entbinden möchten, wissen, dass die Vagina dort nach der Geburt nicht wieder verschlossen wird. Zudem wird jedes Paar über die Folgen der FGM für die frischgebackene Mutter aufgeklärt. Auch die Folgen von FGM für junge Mädchen sowie die Strafbarkeit aufgrund der äthiopischen Gesetzgebung werden thematisiert - insbesondere dann, wenn die Schwangere ein Mädchen erwartet, das ebenfalls ein Opfer von FGM werden könnte.
Die Literatur findet sich unter www.saez.ch $\rightarrow$ Aktuelle Ausgabe oder $\rightarrow$ Archiv $\rightarrow$ 2019 $\rightarrow$ 17-18.
Dass das ganze Vorgehen immer mit dem Partner der Schwangeren abgestimmt wird, ist enorm wichtig. «Denn nur gemeinsam mit allen Beteiligten wird es möglich sein, dem grausamen Ritual ein Ende zu bereiten», erläutert Annette Nehberg. «Dazu gehört insbesondere, dass es uns gelungen ist, höchste Geistliche des Islam als Partner zu gewinnen.» FGM ist Sünde und unvereinbar mit den höchsten Werten des Islam. «Fälschlicherweise wird FGM noch immer mit heiligen Schriften oder religiösen Pflichten begründet und gerechtfertigt», ergänzt Annette Nehberg.

Dank der Arbeit von TARGET e.V. ist die Zahl der Genitalverstümmelungen mittlerweile rückläufig und FGM seit Jahren sowohl nach Landes- als auch nach Stammesrecht verboten. Doch Unwissenheit und die Fesseln der Tradition sorgen dafür, dass das grausame Ritual noch immer praktiziert wird. Ein weiterer Fokus der Menschenrechtsorganisation liegt deshalb nun darauf, die Anwendung der Gesetze voranzutreiben und die Strafverfolgung zu begleiten.

\section{Wieder im Einsatz}

Auch Roswitha Hausdorf und Ludwig Kronpaß wollen das Projekt weiter unterstützen und den Mädchen und Frauen der Afar unnötiges Leid ersparen. «FGM ist eine echte, wenn auch nur punktuelle, humanitäre Katastrophe, weil jungen Mädchen und Frauen körperlich und seelisch sehr grosser Schaden zugefügt wird», sagt Ludwig Kronpaß. «Und bei diesem Projekt gefällt mir ganz besonders, dass TARGET sich vor Ort ganz gezielt um diese eine Thematik kümmert.» Bald wird er wieder für vier Wochen in die Danakilwüste reisen. «Ich freue mich sehr auf diesen Einsatz, der Flug ist schon gebucht.»

\section{Bildnachweise}

S. 627, Bild Mitte und rechts: Ludwig Kronpaß; alle übrigen Fotos: ( ) TARGET e.V. 